\title{
Toward Automatic Motor Condition Diagnosis
}

\author{
J. Ilonen ${ }^{1}$, P. Paalanen ${ }^{1}$, J.-K. Kamarainen ${ }^{1}$, T. Lindh ${ }^{2}$, J. Ahola ${ }^{2}$, \\ H. Kälviäinen ${ }^{1}$, and J. Partanen ${ }^{2}$ \\ 1 Department of Information Technology, \\ 2 Department of Electrical Engineering, \\ Lappeenranta University of Technology, \\ P.O.Box 20, FI-53851, Lappeenranta, Finland
}

\begin{abstract}
In this study a method for automatic motor condition diagnosis is proposed. The method is based on a statistical discriminance measure which can be used to select the most discriminative features. New signals are classified to either a normal condition class or a failure class. The classification can be done traditionally using training examples from the both classes or using only probability distribution of the normal condition samples. The latter corresponds to typical situations in practice where the amount of failure data is insufficient. The results are verified using real measurements from induction motors in normal condition and with bearing faults.
\end{abstract}

\section{Introduction}

Automatic condition monitoring and diagnosis are important in modern industrial installations where a high degree of automation is desired. Automatic monitoring is needed to detect and recognize system faults, such as motor failures, where an early warning could prevent escalation of the problem. This is the case for example in motor bearing damage detection $[9,11]$. There is a variety of sensor and control signals available, but current practice lacks general monitoring methods.

In this study, a diagnosis method is proposed to find discriminative regions, bands, from frequency content of two classes of signals (normal/failure) and to classify new measurements to these classes. The proposed method is useful in cases where there are measurements, but the physical characteristics of failures are not known. It is evident that a sufficient amount of measurements from the both classes are needed in order to find the most discriminative features, but the case where there are measurements mainly from the normal class is of special importance. In practice, measurements from failure conditions may not be sufficient due to economical reasons or difficulties to realize them.

In our experiments the proposed method was successfully applied to detection of bearing damages in induction motors based on their stator current content. 


\section{Discriminative Measures}

\subsection{Feature Extraction}

A set of features must be selected and among them the best ones can be found by comparing their discriminative power.

Two sets of signals, $x_{k}(t)$ and $y_{k}(t)$, represent examples from two classes, $C_{1}$ and $C_{2}$, respectively. The sub-index $k$ denotes a measurement number, $k=$ $0,1, \ldots, N_{1}-1$ for $C_{1}$ and $k=0,1, \ldots, N_{2}-1$ for $C_{2}$. It is assumed that the signals are measured during a stationary system mode, i.e., system parameters such as rolling speed and load are constant. Now, the discriminative information should be present at some frequency band and it is sufficient to apply a bandpass filter $\psi(t)$. In a stationary system mode the time information can be ignored and a global feature, such as a power spectrum

$$
\int_{-\infty}^{\infty}\left|\psi(t) * x_{k}(t)\right|^{2} d t
$$

can be utilized. The selection of the best features is reduced to finding the optimal values for the central frequency $f$ and bandwidth $\gamma$ of a band-pass filter. The normalized Gabor filter

$$
\psi(t)=\frac{|f|}{\gamma \sqrt{\pi}} e^{-\left(\frac{f}{\gamma}\right)^{2} t^{2}} e^{j 2 \pi f t}
$$

where $f$ denotes the central frequency and $\gamma$ controls the spatial sharpness and frequency bandwidth can be used as the band-pass filter.

\subsection{Statistical Measures for Discriminative Power}

If there are several frequency bands where the contents of the classes $C_{1}$ and $C_{2}$ are dissimilar, then the band where the separation of the classes is most evident should be selected. The first-order statistics approach is not sufficient since it simply selects the frequency band where the distance between the expectations is largest, but neglects the variance information, and thus, a significant overlap of the class probabilities may exist $[5,4]$. In the second-order statistics typically an assumption must be made about forms of probability distributions $p_{x}(n)$ and $p_{y}(n)$ which describe the spread of features of the both classes.

It was assumed that the features are extracted from signals measured during

a constant operation mode where variance in the measurements is supposed to be caused by a large number of unknown independent sources. It can be thus assumed that the form of the probability distributions is Gaussian. Now, both classes are uniquely defined by their expectations $\left(\mu_{x}, \mu_{y}\right)$

$$
\mu_{\xi}=E\left[\int_{-\infty}^{\infty}\left|\psi(t) * \xi_{k}(t)\right|^{2} d t\right]
$$


and variances $\left(\sigma_{x}^{2}, \sigma_{y}^{2}\right)$

$$
\sigma_{\xi}^{2}=E\left[\left(\int_{-\infty}^{\infty}\left|\psi(t) * \xi_{k}(t)\right|^{2} d t-\mu_{\xi}\right)^{2}\right] .
$$

For Gaussians Fisher's discriminant ratio $(F D R)$ can be used to measure the distance between the distributions [7]

$$
F D R\left(p_{x}(n), p_{y}(n)\right)=\frac{\left(\mu_{x}-\mu_{y}\right)^{2}}{\sigma_{x}^{2}+\sigma_{y}^{2}} .
$$

Using the divergence measure in (5) the discriminative energy function can be defined as

$$
E=\frac{1}{2}\left(\frac{\left(\mu_{x}-\mu_{y}\right)^{2}}{\sigma_{x}^{2}+\sigma_{y}^{2}}\right)^{2} .
$$

In this case it was possible to establish a formula for the discriminative energy, but in a more general cases other techniques, such as the AdaBoost boosting algorithm [2], can be used to select features.

\subsection{Verifying Gaussianity}

Since the measure is based on the Gaussianity assumption it must be verified. Kurtosis is a fourth-order statistic and has been used to examine the Gaussianity of distributions. A normalized kurtosis with adjusting for bias is [3]

$$
k(x)=\frac{E\left\{(x-m)^{4}\right\}}{\left[E\left\{(x-m)^{2}\right\}\right]^{2}}-3
$$

where $m$ is the mean of $x$. The kurtosis is zero for a Gaussian distribution. Distributions having a negative kurtosis are subgaussian, e.g., uniform or multimodal distributions, and those with a positive kurtosis are supergaussian. A typical supergaussian distribution has a sharp peak and long tails.

\subsection{Bayesian Classification and Confidence}

Using the discriminative energy function (6) the frequency $f$ and bandwidth $\gamma$ of the band-pass filter in (2) can be optimized for features in (1). Single or several frequencies can be selected.

Since the normal distribution of features was assumed it is straightforward to establish estimates for means and variances and to classify new signals using the Bayesian decision making which is based on probability theory and the principle of choosing the most probable or the lowest risk (expected cost) option [10].

In this study we wanted to stress also the issue that quality or number of failure measurements is not sufficient or does not cover all failure states. In that case the classification should be based only on the probability distribution of normal condition measurements. This requirement can be established using an equiprobability surface where features inside the surface are classified as normaland outside as failure. The confidence value which defines the surface must 
be selected manually or it can be computed from the measurements using rank order statistics, i.e., by selecting a fractile of training data which is covered by the smallest probability mass inside the surface. Algorithm 1 computes the probability limit value which can be used in classification. The confidence measure is more thoroughly examined from theoretical point of view in [6].

\section{Algorithm 1. Probability limit $p_{\text {eq }}$ using fractile}

1: Estimate the probability distribution $p$ for Class $C_{1}$.

2: Compute probability values $\bar{p}=p_{i=1, \ldots, N_{1}}$ for all $N_{1}$ samples of Class $C_{1}$.

3: $\bar{s}=\operatorname{sort}(\bar{p})\{$ Sort values in descending order $\}$.

4: $p_{\text {eq }}=s$ (fractile $\left.* N\right)$ \{ Select probability limit (edge of equiprobability surface) based on fractile $\}$.

\section{$3 \quad$ Experiments}

\subsection{Induction Motor Bearing Damages}

Induction motors have been a widely studied subject of the condition monitoring $[11,1]$. An important sub-category of induction motor failures are bearing damages, which can be detected from vibration, acoustic noise, temperature, or stator current signals. Bearing damages are attractive for evaluating the proposed method since characteristic frequencies of damage appearance can be analytically solved and compared to automatically found frequencies.

The exact bearing fault characteristic frequencies calculated from the bearing geometry are valid only for ideal bearings. In practice the rolling elements also slide in addition to rotation, and thus, both in literature and practice approximate equations are often used [8]. The approximate equations for inner and outer race defects are

$$
f_{o}=0.4 N f_{r} \text { and } f_{i}=0.6 N f_{r}
$$

where $N$ is the number of balls or rollers and $f_{r}$ is the rotational speed of the rotor.

Stator current is a potential source for detecting faults since rotor eccentricity causes changes to the current. It should be noted that there are always eccentricities in the rotors, but the eccentricities change in the case of a fault. Bearing defects establish new current components which are present at the frequencies

$$
f_{i b}=\left|f \pm m \cdot f_{v b}\right|, m=1,2,3, \ldots
$$

where $f$ is the supply frequency and $f_{v b}$ is the characteristic frequency of vibration caused by the fault [9].

\subsection{Bearing Damage Detection Based on Stator Current}

The stator current data consisted of stator current signals measured from motors in a normal condition $\left(C_{1}\right)$ and motors with a bearing damage $\left(C_{2}\right)$. The measurements contain two cases: no load connected to motors and with a full load. 
For motors with no load discriminative energy $E$ and classification results are presented in Fig. 1. In this case discriminative energy had its maximum near the first harmonic $(202 \mathrm{~Hz})$ of the characteristic frequency $(101 \mathrm{~Hz})$. Also the both classification schemes had the maximal accuracy at the same frequency band.

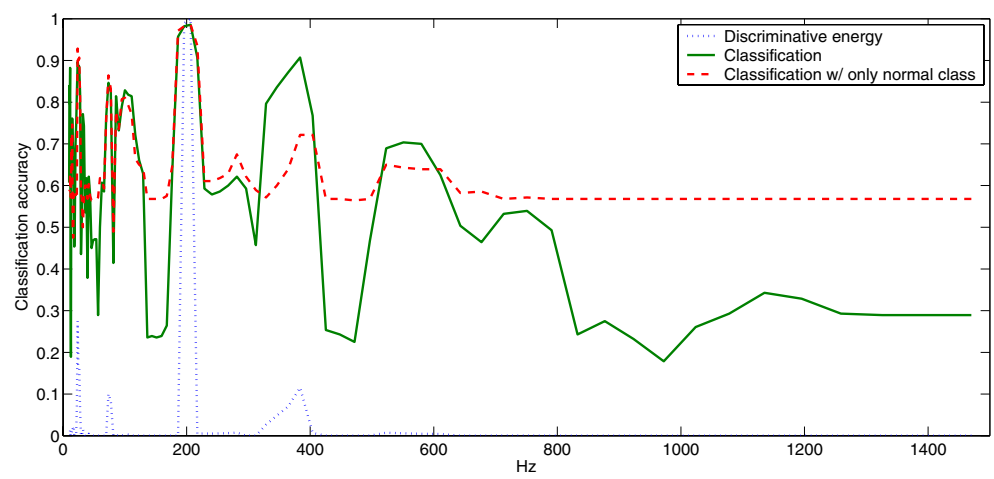

Fig. 1. Discriminative energy and classification accuracies for motors with no load

For motors with a full load results are shown in Fig. 2. This was a more difficult situation since full load caused various disturbances, but still, the characteristic frequency $(101 \mathrm{~Hz})$ and some of its harmonics contained discriminative information. Classifications succeeded on the same frequencies, but due to disturbances the accuracy decreased.

In these experiments the classification was performed using the Bayesian decision making, which requires examples from the both classes, and using the fractile based limit (Algorithm 1), when failure measurements are not needed. The fractile was set to $100 \%$ which means that values within a confidence region that covers the whole training set are accepted as normal condition and all others

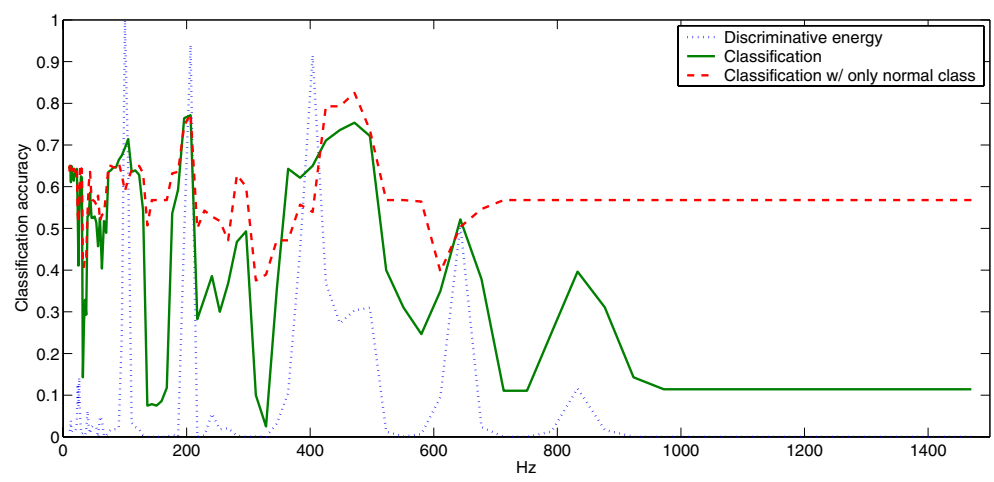

Fig. 2. Discriminative energy and classification accuracies for motors with full load 
classified as a failure. In the both schemes practically the same classification accuracy was achieved at the most discriminative frequencies.

Comparative Results. The experiments were repeated by utilizing the characteristic frequencies in (8)-(9) as reported by Yazici and Kliman in [11]. For the characteristic frequencies and our approach the results are shown in Table 1. Classification was done using the three highest peaks of $E$, both separately and combined, and compared to the classification results with the characteristic frequencies. Characteristic frequencies provided an accuracy of $97.5 \%$ correct classification for a motor with no load. The most discriminative frequency of $E$ provided the same accuracy, but $100 \%$ accuracy was achieved with a combination of the three most discriminative frequencies. For a motor with a full load classification with the characteristic frequencies provided an accuracy of only $66.8 \%$ while the three most discriminative frequencies provided a slightly better classification result, $72.1 \%$.

Table 1. Classification results using calculated characteristic frequencies and three most discriminative frequencies by $E$

\begin{tabular}{r|rr|rr} 
& \multicolumn{2}{|c|}{$\begin{array}{c}\text { No load } \\
\text { Freq. Correct }\end{array}$} & \multicolumn{2}{c}{ Full load } \\
& Freq. Correct \\
\hline Char. freq. & & $97.5 \%$ & & $66.8 \%$ \\
\hline 1st peak & $206.3 \mathrm{~Hz}$ & $97.5 \%$ & $100.1 \mathrm{~Hz}$ & $69.3 \%$ \\
2nd peak & $23.6 \mathrm{~Hz}$ & $87.9 \%$ & $206.3 \mathrm{~Hz}$ & $77.9 \%$ \\
3rd peak & $383.6 \mathrm{~Hz}$ & $91.4 \%$ & $403.9 \mathrm{~Hz}$ & $68.6 \%$ \\
Combined & & $100.0 \%$ & & $72.1 \%$ \\
\hline
\end{tabular}

Utilizing the most discriminative frequencies by $E$ a better accuracy was achieved than with the computed characteristic frequencies as used in literature. It seems that some of the harmonics include noise which harms the classification.

\subsection{Verifying Gaussianity}

The Fisher discriminant is based on the Gaussian probability densities. For other types densities must be explicitly estimated, for example, by using Gaussian mixture models, and the Fisher discriminant must be replaced, for example, with the Kullback-Leibler divergence. The experiments were based on the Gaussianity assumption, and thus, its validity is demonstrated.

Gaussianity was measured with the kurtosis in (7). Kurtoses of the two classes of measurements are shown in Fig. 3(a). At certain frequencies the kurtosis deviates significantly from zero and for those frequencies also the divergence measures fail and the classification cannot provide accurate results. However, by inspecting feature values at locations where discriminative energy is maximal one can ob- 
serve a kurtosis close to zero and also good a separation of the two classes (Fig. 3(b)).

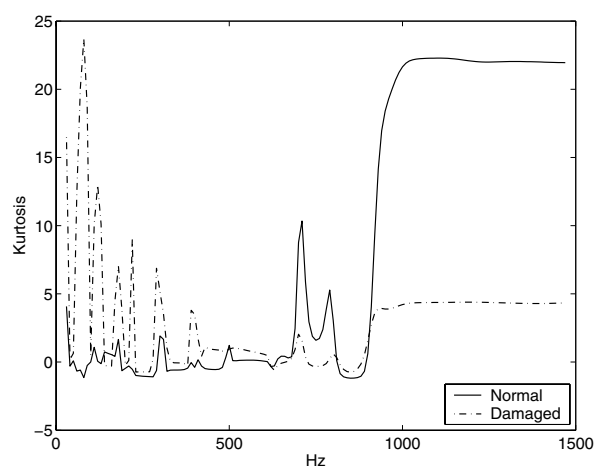

(a)

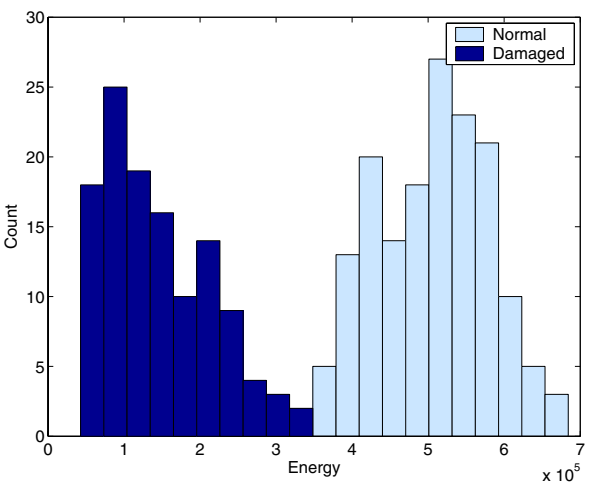

(b)

Fig. 3. (a) Kurtosis of features; (b) feature distributions at $200 \mathrm{~Hz}$ (approx. Gaussian)

\section{Discussion}

In this study, an automatic motor condition diagnosis was studied and methods to automatically select the most discriminative features and to classify new signals were examined. In addition, the case where the amount of failure condition measurements is not sufficient was considered.

In the experiments with the real data the provided measure $E$ was able to find discriminative frequency bands which coincided with the bearing damage fault characteristic frequencies and their harmonics. The classification also succeeded on the same frequencies confirming their validity. The classification based on samples from only the normal class performed equally as compared to the classification with data from the both classes. This is an important result since all possible failure types can rarely be covered in training data.

There is still room for certain improvements which will be addressed in the future to move toward general condition monitoring methods. The stationary assumption can be relaxed by segmenting the signal to stationary parts, the Gaussianity limitation can be overcome by using Gaussian mixture models and the Kullback-Leibler divergence measure. Furthermore, other types of feature selectors can be used, such as the AdaBoost boosting algorithm.

\section{Acknowledgements}

Academy of Finland (project 204708) and European Union (TEKES/EAKR project 70056/04) are acknowledged for financial support. 


\section{References}

1. M.E.H. Benbouzid, M. Vieira, and C. Theys. Induction motors' faults detection and localization using stator current advanced signal processing techniques. IEEE Transactions on Power Electronics, 14(1):14-22, 1999.

2. Y. Freund and R. E. Schapire. A decision theoretic generalization of on-line and an application to boosting. Journal of Computer and System Sciences, 55(1):119-139, 1997.

3. A. Hyvärinen, J. Karhunen, and E. Oja. Independent Component Analysis, chapter 2.7. John Wiley \& Sons, Inc., 2001.

4. J.-K. Kamarainen, V. Kyrki, T. Lindh, J. Ahola, and J. Partanen. Statistical signal discrimination for condition diagnosis. In Proceedings of the Finnish Signal Processing Symposium, pages 195-198, Tampere, Finland, 2003.

5. T. Lindh, J. Ahola, J.-K. Kamarainen, V. Kyrki, and J. Partanen. Bearing damage detection based on statistical discrimination of stator current. In Proceedings of the 4th IEEE International Symposium on Diagnostics for Electric Machines, Power Electronics and Drives, pages 177-181, Atlanta, Georgia, USA, 2003.

6. P. Paalanen, J.-K. Kamarainen, J. Ilonen, and H. Kälviäinen. Feature representation and discrimination based on Gaussian mixture model probability densities practices and algorithms. Research Report 95, Department of Information Technology, Lappeenranta University of Technology, 2005.

7. D.W. Peterson and R.L. Mattson. A method of finding linear discriminant functions for a class of performance criteria. IEEE Transactions on Information Theory, 12(3):380-387, 1966.

8. R.L. Schiltz. Forcing frequency identification of rolling element bearings. Sound and Vibration, pages 16-19, 1990.

9. R.R. Schoen, T.G. Habetler, F. Kamran, and R.G. Bartfield. Motor bearing damage detection using stator current monitoring. IEEE Transactions on Industry Applications, 31(6):1274-1279, 1995.

10. S. Theodoridis and K. Koutroumbas. Pattern Recognition. Academic Press, 1999. ISBN 0-12-686140-4.

11. B. Yazici and G.B. Kliman. An adaptive statistical time-frequency method for detection of broken bars and bearing faults in motors using stator current. IEEE Transactions on Industry Applications, 35:442-452, 1999. 